EDITORIAL

\title{
What is Adequate Surgical Experience for Junior Neurosurgical Residents?
}

Can. J. Neurol. Sci. 2010; 37: 723-724

\author{
Fallah et al. "Surgical Activity of First-Year Canadian \\ Neurosurgical Residents". 1
}

The aim of this study was to assess the variability in surgical experience of first-year Canadian neurosurgical trainees through self-report survey.

Nine PGY1 neurosurgical residents surveyed provided a three-month operative log of their surgical experience. The median number of operative cases was 66 and types of surgery included intracranial hemorrhage, cerebrospinal fluid diversion, intracranial tumors and cervical/lumbar spine pathology. In 12 of these cases, the trainee undertook a primary surgeon role and in 53 cases, the trainee's role was that of an assistant surgeon. The trainees most often functioned as the primary surgeon in cerebrospinal fluid diversion cases and surgery for intracranial hemorrhage.

This study is novel as it is one of the first analyses undertaken to attempt to accurately document operative experiences from a group of junior neurosurgical residents across Canada.

The authors identify a critical concept that has become prevalent in the neurosurgical community when they describe the inevitable need to describe training based on "competency" rather than "time-based". The assessment of competency for surgical technical ability is unique. Clearly a certain number of operations are required to gain competency, however this number may differ for each individual trainee. The authors correctly point out the belief the number of yearly cases to reach neurosurgical competency approximates 250. Although this predicted value serves as a foundation for competency, it is evident that other metrics must be considered as well. These would include the level of difficulty or rarity of the case and the degree of surgical supervision and/or simulation that occurred. ${ }^{2}$ The question remains as to what the optimal benchmarks are to assess adequate surgical progression during a neurosurgical residency program?

Furthermore, the authors clearly recognize some of the limitation of their study as outlined in their statements:

\begin{abstract}
"Over $50 \%$ of those studied were from the same residency program. The interpretation of who is a "primary surgeon" is vulnerable to bias. The study is based on a small snapshot of time".
\end{abstract}

The paradigm of neurosurgical residency training is changing within Canada. These changes are complicated by many factors including a generational shift with different life philosophies, newly implemented rules related to acceptable length of working hours, expectations for assessment of CanMeds competencies, appropriate length of training and subspecialty development.
Within the CanMeds competency of "medical expert", program directors have a number of assessment tools that can be employed. These tools are necessary to assess two main facets of neurosurgical training, the acquisition and retention of medical knowledge and the development of surgical skill and competency.

In order to assess surgical competency, many programs have instituted a resident based operative log system that can be used to assess overall operative statistics. Although labor-intensive, this platform hopefully can be used to identify "gaps" in training such that the trainee's operative experience can be tailored to fill these "gaps" by the end of their residency. Thus, this type of approach can be supported as being an important first step in quantifying the surgical experience our residents are gaining and to hopefully reveal any voids that may exist.

The authors also touch on the "elephant in the room". Five of the nine residents routinely stayed post-call to operate. These residents reported higher surgical volumes and most stayed postcall to enhance their operative experience.

Work hour rules have been developed to protect both patients and physicians. These rules are variable in the world and even in Canada. Currently in Denmark, a 37-hour work week exists. The European Working Time Directive for trainees have adopted a 48-hour work week while an 80-hour work week exists in the United States. Within Alberta, resident emergency medicine physicians are not allowed to complete more than 60-hours of shift work a week. Our neurosurgical residents are instructed to abide by the call frequency ( $1 / 3$ in house call and $24+2$ hours work rule) but I often find that this rule functions sometimes more like a guideline.

The issue of whether or not these rules have impaired surgical training are mixed. Some recent studies have shown minimal operative volume experience changes for senior residents but more dramatic changes in experience for junior residents. ${ }^{3}$ Others have demonstrated that senior resident involvement in "take back" cases have remarkably decreased. ${ }^{4}$ Other surrogate markers for the quality of trainees such as the inservice training scores for the American Board of Surgery have improved with work hour restrictions. ${ }^{5}$ What further confounds the arguments around work hour rules and surgical experience is the revelation that actual operating time may only represent $20 \%$ of a surgical resident's work day.

These issues may and I suspect will create the impetus for us to improve the quality of the educational experience of our residents. Many of us, including myself, are realizing that we have only received rudimentary instruction on how to teach something as complex as clinical neurosurgery. ${ }^{6}$ In the current climate of surgical education, it behooves the surgical educator to look beyond the 42-months of clinical neurosurgery that is 
required and develop a more concrete novel assessment package of technical expertise. The article by Fallah et al is an important first step in the understanding of the surgical experience we are giving our junior trainees. ${ }^{1}$
Vivek Mehta University of Alberta Edmonton, Alberta, Canada

\section{REFERENCES}

1. Fallah A, Ebrahim S, Haji F, et al. Surgical activity of first-year Canadian neurosurgical residents. Can J Neurol Sci. 2010;37(6): 855-60.

2. Reznick RK, MacRae H. Teaching surgical skills - changes in the wind. N Eng J Med. 2006;355:2664-9.

3. Jackson GP, Tarpley JL. How long does it take to train a surgeon? BMJ. 2009;339:1062-4

4. Feanny MA, Scott BG, Mattox KL, Hirshberg A. Impact of the 80hour work week on resident emergency operative experience. Am J Surg. 2005;190:32-329.

5. Vetto JT, Robbins D. Impact of the recent reduction in working hours (the 80 hour work week) on surgical resident cancer education. J Cancer Educ. 2005;20:23-7.

6. Omahen D. The 10,000 hour rule and residency training. CMAJ. 2009;180:12. 